\title{
A Consistent Design Methodology for Wireless Embedded Systems
}

\author{
P. Belanović \\ Institute for Communications and RF Engineering, Vienna University of Technology, Gusshausstrasse 25/389, \\ 1040 Vienna, Austria \\ Email:pbelanov@nt.tuwien.ac.at

\section{B. Knerr} \\ Institute for Communications and RF Engineering, Vienna University of Technology, Gusshausstrasse 25/389, \\ 1040 Vienna, Austria \\ Email: bknerr@nt.tuwien.ac.at

\section{Holzer} \\ Institute for Communications and RF Engineering, Vienna University of Technology, Gusshausstrasse 25/389, \\ 1040 Vienna, Austria \\ Email:mholzer@nt.tuwien.ac.at
}

\section{G. Sauzon}

Infineon Technologies, St.-Martin-Strasse 53, 81669 Munich, Germany

Email: guillaume.sauzon@infineon.com

\author{
M. Rupp \\ Institute for Communications and RF Engineering, Vienna University of Technology, Gusshausstrasse 25/389, \\ 1040 Vienna, Austria \\ Email: mrupp@nt.tuwien.ac.at
}

Received 29 January 2004; Revised 15 February 2005

\begin{abstract}
Complexity demand of modern communication systems, particularly in the wireless domain, grows at an astounding rate, a rate so high that the available complexity and even worse the design productivity required to convert algorithms into silicon are left far behind. This effect is commonly referred to as the design productivity crisis or simply the design gap. Since the design gap is predicted to widen every year, it is of utmost importance to look closer at the design flow of such communication systems in order to find improvements. While various ideas for speeding up designs have been proposed, very few have found their path into existing EDA products. This paper presents requirements for such tools and shows how an open design environment offers a solution to integrate existing EDA tools, allowing for a consistent design flow, considerably speeding up design times.
\end{abstract}

Keywords and phrases: single system description, virtual prototyping, system on chip, automatic verification.

\section{INTRODUCTION}

Complexity of modern communication systems, particularly in the wireless domain, grows at an astounding rate. This rate is so high that the demand of algorithmic complexity now significantly outpaces the growth in available complexity of underlying silicon implementations, which proceeds

This is an open access article distributed under the Creative Commons Attribution License, which permits unrestricted use, distribution, and reproduction in any medium, provided the original work is properly cited. according to the famous Moore's law [1], that is, available complexity doubles every 16 to 18 months. Furthermore, algorithmic complexity even more rapidly outpaces design productivity, expressed as the average number of transistors designed per staff/month $[2,3,4]$. In other words, current approaches to wireless embedded systems design are proving inadequate in the struggle to keep up with system complexity. This problem, first introduced as design productivity crisis in 1999 by Sematech (www.sematech.org), is nowadays well known under the name the productivity gap or design gap. 
Many concepts so far have been proposed to solve the two problems: (1a) introducing more parallelism and reusing intellectual property (IP), (1b) special hardware (HW) accelerators, and (1c) trading design time against complexity and (2a) virtual prototyping (VP), (2b) optimal HW/SW codesign, (2c) cosimulation on different design levels, and (2d) using new languages to overcome the fragmentation of the design process, to name the most common ones. ${ }^{1}$ While some commercial EDA tools adopted a few of such solutions as their tool features, no tool allows for all of them.

Before a consistent proposal is presented, addressing adequately all of these features, the advantages and disadvantages of each of them will be discussed briefly in Section 2 . This discussion also allows for defining the requirements of a future solution. This paper presents an integrated design methodology and its implementation in the form of an open design database supporting all of the requirements defined in Section 2 including a single system description (SSD) by a database approach as well as automatic VP generation. Section 3 presents the details of such a design database $[5,6]$. An SSD allowing to convert designs to various design levels (Section 3.1) and the binding of several domain specific modeling languages (DSML) into this integrated design methodology are described (Section 3.2). Included in the integration are well-established languages, such as VHDL, as well as notable new system-level modeling languages like SystemC. Also the development environment for algorithms COSSAP from SYNOPSYS as well as its used C derivative programming language GenericC is supported. The inclusion of virtual prototyping in $\mathrm{C}++$ is demonstrated in Section 4 supporting rapid HW/SW co-design. With the proposed design methodology, soft guidelines along the design path ensure that all major steps can be performed automatically, speeding design time up considerably. In Section 5 consistent testing at all abstraction levels of the design process derived automatically from the SSD is demonstrated in detail. Finally, an industry-developed wireless communication design (UMTS receiver for a cellular phone) is presented in Section 6, utilizing the new design flow and resulting productivity improvements are reported.

\section{REQUIREMENTS FOR SHORT DESIGN CYCLES}

While possible solutions for dealing with the complexity as well as the productivity gap were quickly listed in the introduction, in this section we will discuss more thoroughly the various solutions and their impact on the design.

More parallelism and reusing IP seems to be a simple answer to solve the complexity problem. However, more parallelism is equivalent to more chip area and thus more power demand. In particular in the field of mobile wireless communication, this is not an acceptable solution. Reusing IP does only help speeding up design processes once the IP has

\footnotetext{
${ }^{1}$ In our notation, (1a), (1b), and (1c) are solutions for the complexity gap while (2a), (2b), (2c), and (2d) denote solutions for the productivity gap.
}

been created. Thus, at the beginning of a new product design, there simply is no IP that can be reused. Also building IP in a reusable fashion, that is, parameterised, costs considerably more time than building a block based on fixed constraints. Unfortunately, time-to-market is very decisive in wireless modem design: launching six months early triples profits, six months late results in breaking even [7].

Special HW accelerators are currently offered by many start-up companies especially for the $3 \mathrm{G}$ market. Sandbridge (www.sandbridgetech.com), Intrinsity (www.intrinsity. com), Tensilica (www.tensilica.com), and Adelante Technologies (www.adelantetech.com) are typical examples offering fixed or configurable HW accelerators for a typical DSP structure tailored to support the needs of a $2.5 \mathrm{G}$ and 3G manufacturer. Most impulses to offer even more flexible structures that can be specified and modified by the designer himself are still coming from universities $[8,9,10]$ and seem to be far from being included in commercial electronic design automation (EDA) tools. Even more advanced tools that are reconfigurable at run time are in an early research stage at universities $[11,12]$ and some are even announced by some start-up companies (www.picochip.com, www.quicksilvertech.com).

Trading design time against complexity has been used in the past in almost every complex design, in particular in those designs based entirely on $\mu \mathrm{Ps}$ and DSPs. When the time-to-market of the product was known to be, say, three years, then Moore's law predicted availability of a four times higher complex processor. Thus, the design engineers simply assumed a processor with such complexity available at the time of product introduction and started the functional integration process for it. By the time the integration process was finished, the processor was available and the product was assembled. In particular, in GSM phone development this process was quite successful, relying simply on the next-generation DSP of a specific family. Once the complexity gap widened, one could still start a design process built on this tradeoff with the drawback that some features would not be offered in the first product, but only a year or two later, when the faster processor would be available. However, now with even shorter time-to-market constraints and a widening complexity gap between demanded and available complexity, this predictive-design method is outdated and can only offer small advantages.

Virtual prototyping $(V P)$ has been introduced in the design process a couple of years ago $[13,14,15,16]$ and was quickly adopted by design houses [17]. The VP consists of a complete description of HW and SW components in a software form that can be simulated on a general platform. It includes in particular an interface description between the various HW and SW entities, allowing to simulate the communication between these blocks (transaction-level modeling). The timing granularity can differ in the model of the VP from untimed (timing is neglected) to cycle true (RTL modeling) descriptions. Also hybrid forms exist like bus-cycle accurate, where the system behaves only cycle true at the bus interface. Since SW development is in general much faster than the corresponding HW development, the VP, notably 
untimed or bus-cycle accurate, implemented is much earlier available than the real-time platform. It thus allows an earlier development of the SW related programs since it is no longer required to wait until the final working HW platform is available. While being a successful instrument over the past years in $2 \mathrm{G}$ and $2.5 \mathrm{G}$ development, the VP generation becomes burdensome in $3 \mathrm{G}$ development. Since the required complexity in $3 \mathrm{G}$ is expected to be 100 to 1000 times higher than in $2 \mathrm{G}$, a fully detailed VP does not only require much more SW design but it also runs very slowly on available standard platforms (like Pentium or Sparc-based computers).

Optimal HW/SW codesign has been a research effort over many years. Heuristics like simulated annealing (SA) used in the COSYMA system [18], genetic algorithm (GA), and tabu search are usually used to tackle the NP complete problem of HW/SW partitioning. A comparison of those algorithms is given in [19]. Even evolutionary algorithms are applied to that problem, which is shown in [20]. Despite those efforts, such solutions are not found in commercial EDA tools. The reason for this seems to be that their assumptions are not sufficiently practical, that is, the required information cannot easily be extracted from the high-level design. Typically, a complete metric for a HW and SW solution is required a priori in order to decide how to optimally partition the design. However, such knowledge is not known beforehand. The implementation effort in $3 \mathrm{G}$ wireless is so high that an a priori realisation of the system would be too expensive.

Simulation times are growing tremendously. In [21] an example is presented, where four seconds of real-time take five minutes of simulation time in $\mathrm{C}$ at the algorithmic design level (assuming processor clock at $150 \mathrm{MHz}$, bus clock at $50 \mathrm{MHz}$ ). If simulated on gate level, the simulation time alone would take 1.25 years. Since testing is typically performed by simulation and cosimulation, testing time is increasing in $3 \mathrm{G}$ designs. Today, about $70 \%$ of development time is spent on verification and testing $[15,22]$. In order to decrease such extensive times, cosimulation on different design levels promises to be a good solution. Since higherlevel models require less simulation time, it is of advantage to run only the function under test on the lower design level while the remaining parts still run on higher levels. Cosimulation has been offered by SYNOPSYS (www.synopsys.com) and CADENCE (www.cadence.com) as a possibility to run instruction-set simulators (ISS) for various DSPs in parallel with their algorithmic design tools COSSAP and SPW, respectively. SYNOPSYS for example offers cosimulation of C (SystemC) and VHDL on their CoCentric System Studio design environment. However, there is no automatic interface generation to connect the $\mathrm{C}$ programs to the VHDL programs and the designer has to write this for each block manually, a time consuming process that is known to be errorprone.

New languages have been proposed very often during the past years depending on the application fields like UML (www.uml.org) by the object management group (OMG) and SDL [23] by the International Telecommunication Union (ITU-T). Even JAVA (www.sun.com) has found some attraction. However, it is not easy to convince chip designers with 15 years of VHDL or VERILOG experience to use a new language just because some other parts of the design team may profit from it. SystemC [24] (www.systemc.org) seems to be a much better approach since $\mathrm{C}$ has been used almost for all design stages, the design engineers being at least familiar with it. The library extension of $\mathrm{C}++$, SystemC v2.0, allows to specify fixed-point arithmetic as well as abstract interfaces between entities. The extension towards HW description follows almost entirely the concept of VHDL, thus allowing VHDL designers to reuse their experience in a slightly different language. However, with the advent of SystemC, not all problems have been solved, SystemC basically offers a uniform language platform on which the design problems can be solved.

A major problem which causes design delays is the fragmentation of the design process. Due to the large scope and extremely heterogeneous nature of modern wireless communication designs, their development suffers from incompatible system descriptions. These descriptions are rewritten by corresponding experts converting them into other, more suitable description forms. The outcome of this is serious communication obstacles between design teams due to different approaches and languages. Also inconsistent verification, lack of design tools for supporting necessary design steps, and difficulties in the discovery and fixing of bugs $[25,26]$ are evident.

These problems arise from the fragmentation of the design process into domain-specific efforts. A formal methodology to keep the design integrated and progress consistent through the development process is missing. In [27] such an integrated environment named POLIS has been reported for typical control-oriented, reactive systems based on high-level languages like ESTEREL [28] and LUSTRE [29], supported by a PTOLEMY [30] environment and based on extended finite state machines (EFSM). Such an environment allows to design a problem in graphical form and to map it onto microcontrollers. There is also related work in the field of model-integrated computing (MIC) including treatment of DSMLs and their integration $[31,32,33]$. An extended approach based on the MIC environment for the integration of different tools is presented in [34]. An open standard called Open Access for the data exchange among IC design tools is driven by the Open Access Coalition (www.si2.org). Within this standard the main focus is on the integration of tools for layout.

Based on this discussion, the requirements of a design method allowing for fast design cycles in wireless chip design are the following.

(1) Support of different design levels at the same time: only through a mixed-level approach, where cosimulation of different abstraction levels is performed, can testing times be brought down.

(2) Translational tools allowing to derive other descriptions automatically: in order to have a consistent process that is not hampered by manual rewrites of one and the same design many times, thus producing errors and slowing down the design process, it is imperative to use translational tools in order to provide consistency and speed. 
(3) A single system description: although translational tools may convert one design description into another, information may get lost by this process. An SSD keeping all information in a database can prevent such a problem. The translational tools thus need to be linked to such an SSD in order to decide what information is needed for a requested transformation. An SSD can guarantee the consistency in the design which is especially important when an error appears at a different design level than it was produced.

(4) A single design language: is quite advisable since design can be performed in terms of refinement steps rather than rewrite or reformulate operations. Refinement ${ }^{2}$ is typically a much more efficient design method and not as error prone. SystemC seems to be a good compromise since ANSI$\mathrm{C}$ has a wide acceptance for the algorithm development, the architectural language elements are very similar to VHDL or VERILOG and thus can be used by designers for architecture definitions as well as for lower design description on RTL. Also, $\mathrm{C}$ as simulation language runs relatively fast in simulations, a further advantage.

(5) An open design environment: in order to save development time, EDA tools should follow an open standard (like VSIA) so that an integrated design environment can be linked to such tools that are most favoured by the design groups. Also, once new tools are available, like tools for automatic floating-point to fixed-point conversion or optimal HW/SW partitioning, these tools can seamlessly be integrated into such environment and do not require slow manual processes. $^{3}$

(6) Virtual Prototyping: speeding up the design process by implementing SW and HW in a parallel manner rather than sequentially is certainly obligatory. A flexible VP design can also support various design levels including platform-based designs. In this context, a VP can insure that refinement techniques are applied, a single description language is used throughout the design, and testing can be performed at different design levels. Although no commercially available VPs are known to the authors, VSIA (www.vsia.org) has defined specification rules for VPs.

\section{INTEGRATED DESIGN METHODOLOGY}

As explained in the previous section, an integrated design methodology is required to work with existing EDA tools. Since the information required for various tools is never identical, a consistent design methodology needs to utilise a so-called SSD, containing all design information. Depending on the applied tool, only part of this information, specific

\footnotetext{
${ }^{2}$ Note that the refinement technique together with the one-code paradigm is one of the crucial elements in rapid prototyping as well [26].

${ }^{3}$ Note that Sematech in its original report in 1999 concluded the following: (1) for every \$1 invested in EDA tools, an additional \$2 to \$5 are spent on integration into the design flow; (2) no EDA vendor or using company can supply all the tools needed today; (3) promote rapid integration of new tools from industry and university research; (4) create chip hierarchical design system technical date (CHDStd) standard. None of these have become reality.
}

to this tool, is presented to the designer. This section explains the details of such an SSD and how it supports various DSMLs.

\subsection{A single system description}

An elaborated solution of an SSD is the implementation in the form of an SQL-DDB $[5,35]$ running on a SUN workstation. A database representation is not bound to specific language constraints and thus offers great flexibility in capturing the miscellaneous aspects of a design. Additional advantages of the DDB approach are fast access, data security by the capability to grant permissions to the developers, a high popularity, as well as compatibility with major database management systems (DBMS) from Microsoft, IBM, Oracle, and the open source DBMS MySQL.

A framework reflecting these obligations is shown in Figure 1. It depicts the DDB surrounded by the required tools each with dedicated interfaces to incorporate the various EDA tools and stays open for incorporating other tools as the empty tool box in the figure indicates. The various design teams provide inputs, such as desired system behaviour and structure, constraints, and tool options. Also, the designers receive outputs, like status of the system description, results of simulations, estimates of hardware costs, timing, and so forth. Typically, the outputs of the database are handed to the tools which present them in form of their GUIs to the designer. Some of the tools supported by the SSD are commercially available, favoured by the various design teams, while others are specially written to perform missing tasks, usually performed manually by designers in the past. As long as some design steps are not covered by available tools, for example HW/SW partitioning, a database modification tool is available, simply allowing the designer to enter manually derived values. The database is thus enriched and the system description is refined on its way to implementation. Note that the database system does not require a specific order of which various tools need to be performed. For example, some designers prefer to perform floating-point to fixed-point conversion after the HW/SW partitioning. As long as the succeeding tool is provided with sufficient information, it can be started. Such open environment has not only the advantage that new commercial tools can be incorporated but it also provides a realistic platform to investigate the performance of new research tools. A possible design flow example is indicated in Figure 1. At first a COSSAP project is read into the SSD. In the second step the content of the database can be investigated over the HTML Visualization. As the last step, a virtual prototype is automatically generated out of the database content.

\subsection{Inclusion of domain-specific modeling languages}

The underlying concept of the translational tools allowing to import designs into the database is two-tiered, consisting of a parser and a scanner, as Figure 2 illustrates. Both parts together establish the system description interface (SDI). The parser is adapted to the EDA tool or the description language and translates the information contained in the code to an XML-based intermediateformat (IF), which has been 


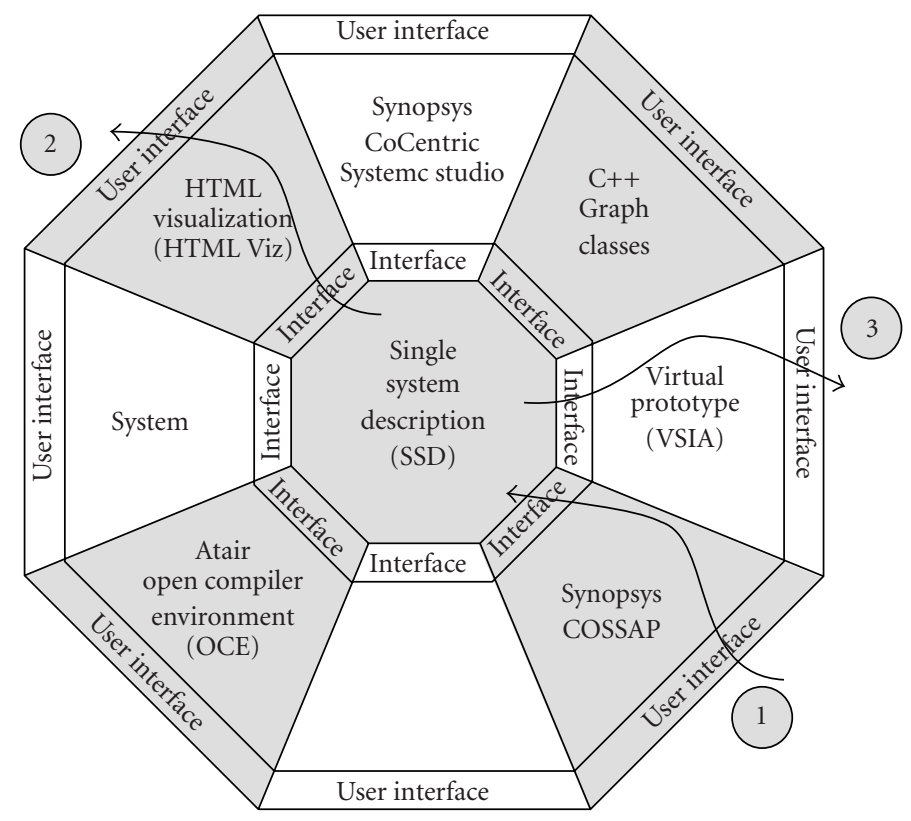

FIGURE 1: Interaction of the single system description with different design tools via interfaces providing a consistent design flow.

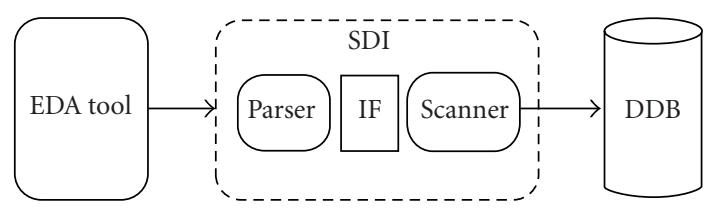

Figure 2: Concept of the system description interface.

especially developed to represent the content of the database in a textual description, containing all relevant information, such as module instances, signal interconnections, and port declarations.

The SSD as the central repository of the consistent design environment has been designed to generally fit system descriptions and also design properties. The system description part supports concepts as modules or entities, their hierarchy, and interconnections. This concept allows to store design languages with concepts of parallel processing and procedures. On the other hand, synchronous reactive languages like ESTEREL cannot directly be mapped to the DDB. The core of the underlying DDB structure is shown in Figure 3.

In addition to such concurrent concepts, sequential parts of system descriptions, such as processes and operation sequences are also supported. The nomenclature of all concepts in the database structure implementing the single system description was strongly inspired by SystemC. All entities that make up the system are instances of modules. These instances form one or more layers of hierarchy. Each of the instances can contain one or more processes. All processes in the system run concurrently. Processes are internally formed by basic blocks. Within such basic blocks sequences of operations are identified. Communication between instances, processes, and operations is achieved through data. Data connecting several instances has several aliases; one within the context of each of the connected instances. An alias has an alias type, such as input, output, in-out port, or internal signal. Data has a data type, such as a signal, variable, or constant. Operation also has an operation type, such as addition $(+)$, multiply-accumulation (MAC), or left bitwise shift $(\ll)$.

Figure 4 shows a graphical representation of a small design written in SystemC. Example_1 which is an instance of Example has inputs (in1, in2, in 3 , in4) and an output (out1). It contains three blocks: two adders (add_1, add_2) and one multiplication (mul_1), and their corresponding processes. The add blocks are connected with the multiplier internally with the signals int 1 and int2. Here, the alias concept is useful to identify these signals with the internal output signals of the add blocks and the input signals of the multiplier. A cut-out of the XML-based IF of this example is presented in Figure 5.

A scanner tool processes this IF and enriches the DDB accordingly. In this simple case the module table holds the names for the used modules Example, add, and mul. Inside of the instances table, Example_1, add_1, add_2, and mul_1 can be found and the alias table stores the ports in_1, in 2 , in $\_3$, in $\_4$, out_1, and the internal signals int 1 and int 2 . The two-stage structure facilitates the development of new interfaces in such a way that the developer of the parser can neglect everything related to database management, that is, adding or deleting modules, removing redundant information, updating of attributes and so forth. This task is fulfilled by the scanner tool, which depends only on the IF and the current contents of the DDB.

As an example, one of the realised SDI implementations is presented, where for example the algorithm design team favours COSSAP as a graphical representation of the design 


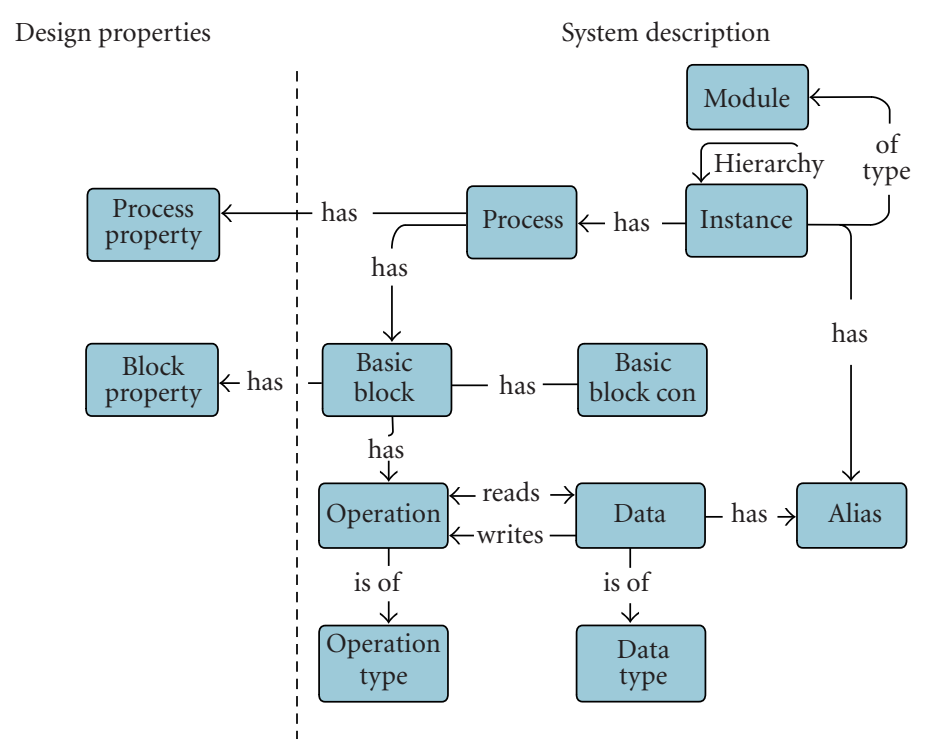

FIgURE 3: The structure of the design database (DDB).

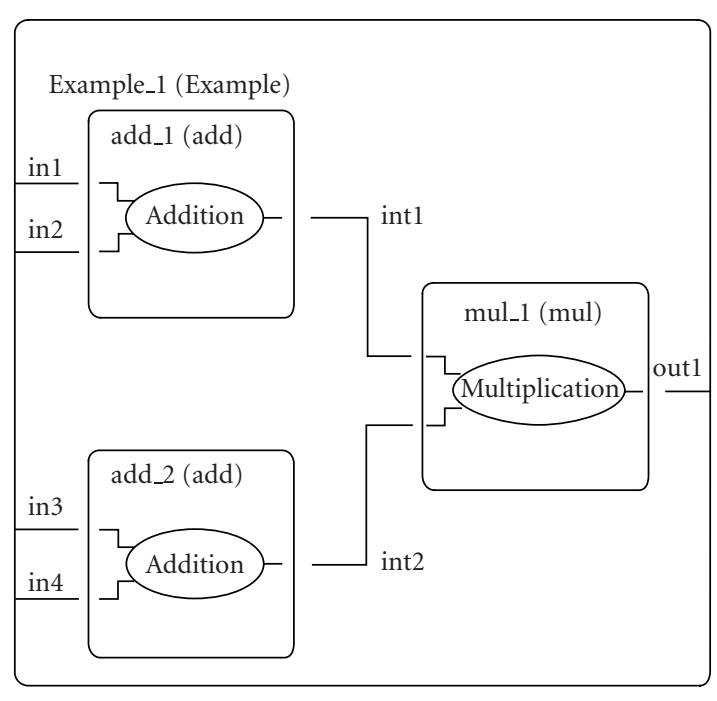

Figure 4: Design example.

at system level. To provide a complete representation of the COSSAP model within the DDB, the information on the architectural structure as well as on the functionality and behaviour of each component is extracted from the COSSAP project. A COSSAP project stores its model architecture in a nonhierarchical way in a file pair named after the top module, suffixed ".v_arc" and ".v_ent." The description language used in these files is VHDL compliant. A parser for VHDL has been implemented to create the IF representation of the model architecture (see left-hand part of Figure 10 further ahead). This parser is based on two open source tools: Flex (www.gnu.org/software/flex/flex.html), a lexical analyzer, and Bison (www.gnu.org/software/bison/bison.html), a parser generator. The scanner, a set of PERL scripts,

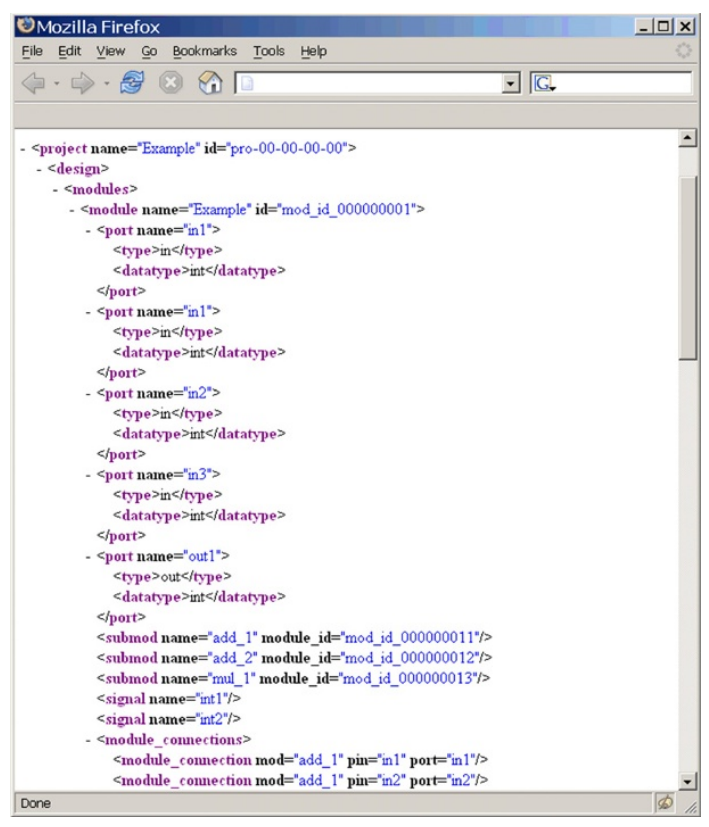

Figure 5: XML-based intermediate format.

processes the achieved IF and adds the information to the DDB. Each component of the COSSAP model has its own GenericC file containing the behavioural model. GenericC is a derivative of $\mathrm{C}$ and proprietary standard of COSSAP. A parser for these files was implemented to make them ANSIC compliant for further processing. The developer of the GenericC files has to adhere to a guideline catalogue, to enable a smooth automatic transition to ANSI-C. These guidelines consist mainly of name conventions and have negligible impact on the COSSAP development routine. In particular, the proposed guidelines did not limit the creativity of 


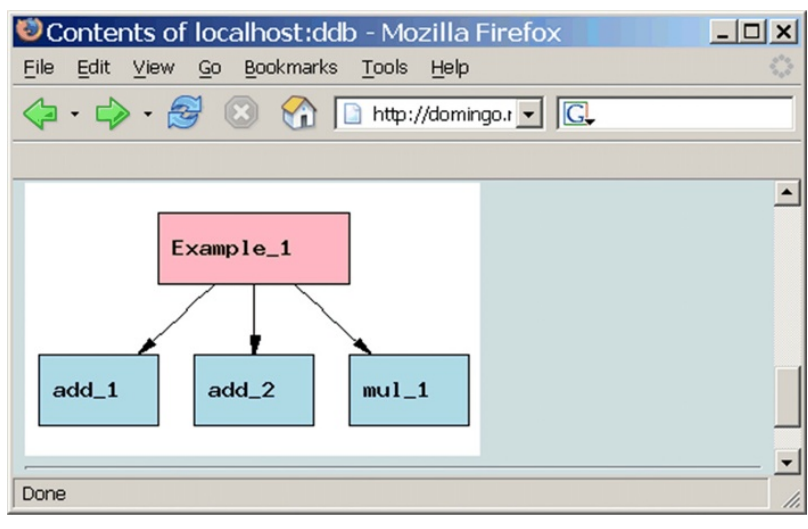

FIgURE 6: HTML visualisation of the database content. Hierarchical view of the modules.

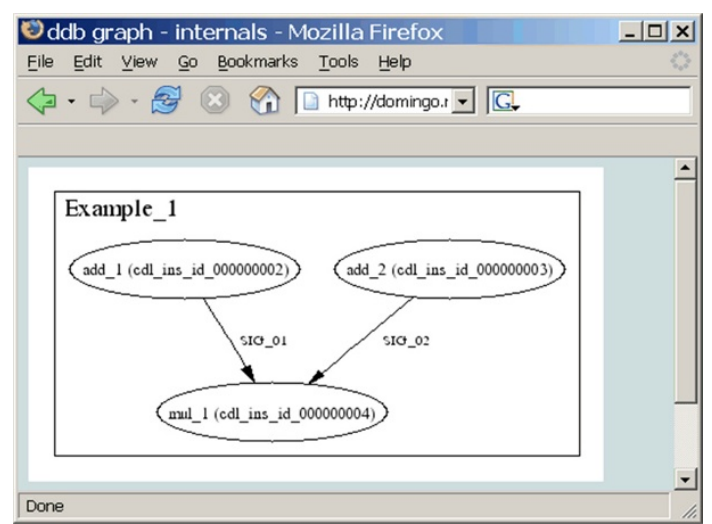

FIGURE 7: HTML visualisation of the database content. Data flow graph of modules.

the algorithmic design team and were thus quickly adopted throughout the algorithmic design team. While other SDIs for the SSD exist (e.g., for reading SystemC v1.0 designs), we will focus on the COSSAP SDI in this paper.

\subsection{HTML visualisation}

As mentioned before, some design steps are not supported by automatic tools yet. To facilitate manual interaction with designer, GUIs for viewing information and changing entries are provided. To support the designers working on the development of the system, whose description resides in the database, with a visual representation of the system, an HTML visualisation over a web browser has been developed. This software program, implemented as a PERL CGI script, provides an easily extendable view of the database contents. Since it is a CGI script, a dynamic view of the system description, immediately reflecting any changes and updates, is provided. Different views of the database content can be represented over a browser. For example, the hierarchy of instances in the system is represented graphically (Figure 6), where it can be seen that the modules add_1, add_2, and mul_1 are instantiated inside the module Example_1. Figure 7 depicts the data flow graph of the modules in Example_1.

\section{VIRTUAL PROTOTYPING}

The HW/SW partitioning process transforms a system-level specification into a heterogeneous architecture composed of hardware and software modules. A software simulation of this simulatable model is called virtual prototype (VP) $[13,14,15,16]$. Generally, the VP reflects a platform-based design (PBD), typically a DSP surrounded by multiple HW accelerators (HA). Those HW accelerators are called VP components if they are used inside a VP simulation. Several properties of abstraction layers are proposed for a VP, as they can be time related (e.g., untimed, timed functional, bus-cycle accurate, cycle true), data related (e.g., floatingpoint and fixed-point representations), and communication related (e.g., synchronous data flow (SDF), transaction-level modeling (TLM) [36], open core protocol international partnership OCP [37]).

A crucial point in the partitioning for a SoC-design is a carefully designed interface, connecting hardware and software parts. Whereas hardware development, and especially its testing, can be done rather independently from the software development, development and testing of the software has to wait until the hardware has been designed. In the case of an application-specific integrated circuit (ASIC), verification cannot start until the first engineering samples have been manufactured (Figure 8).

The VP technique on the other hand enables earlier development and testing of the software. In this technique, SW reflects the behaviour of the HW and implements the HW interface to the SW, as it will be realised later in HW. Such a VP can be implemented faster than the HW itself, because all the HW implementation details specific to the chosen technology can be neglected and high-level description languages can be used instead of hardware description languages (HDLs).

Other advantages of a VP are its capability to serve as a reference (golden) model for the HW accelerators, whose functionality it mirrors at the bus interface as well as to support refinement steps: a first version of the VP can be generated without considering any architecture or timing (untimed). Then, in a next step, the specific HW target platform can be included with a DSP-core element, a bus, and various HW accelerators, which communicate bus-cycle accurate. As a last step, the internal behaviour of the HW accelerators has to be transformed to a cycle-true model. This step is usually called high-level synthesis and is investigated by many research projects like for example in [38] and also adopted in commercially available tools (behavioural compiler from SYNOPSYS). A survey of high-level design transformation techniques is given in [39]. In that sense, VP also supports a refinement-step-based design, which allows a much more consistent forgoing than switching between description languages.

\subsection{On automatic VP generation}

In the following we present an automatic generation method for a VP tailored for platform-based designs, which allows for a further decrease of development time as shown in 


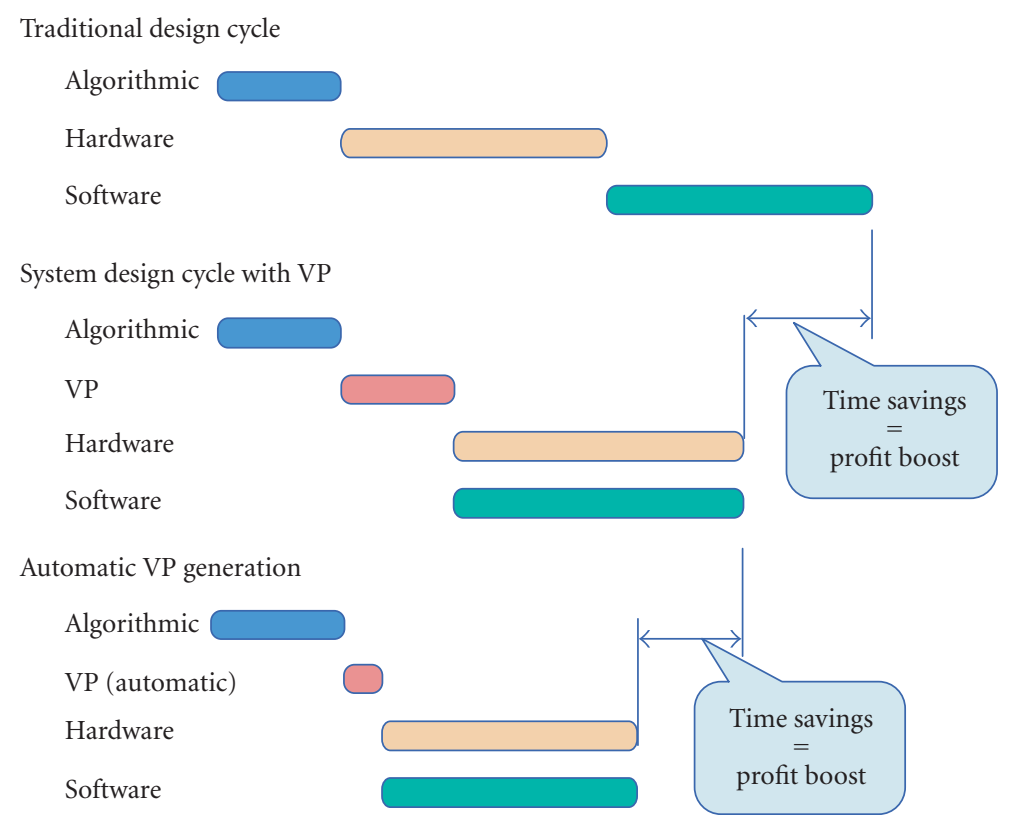

FIGURE 8: Decrease of design time by virtual prototyping and automatic generation of virtual prototypes.

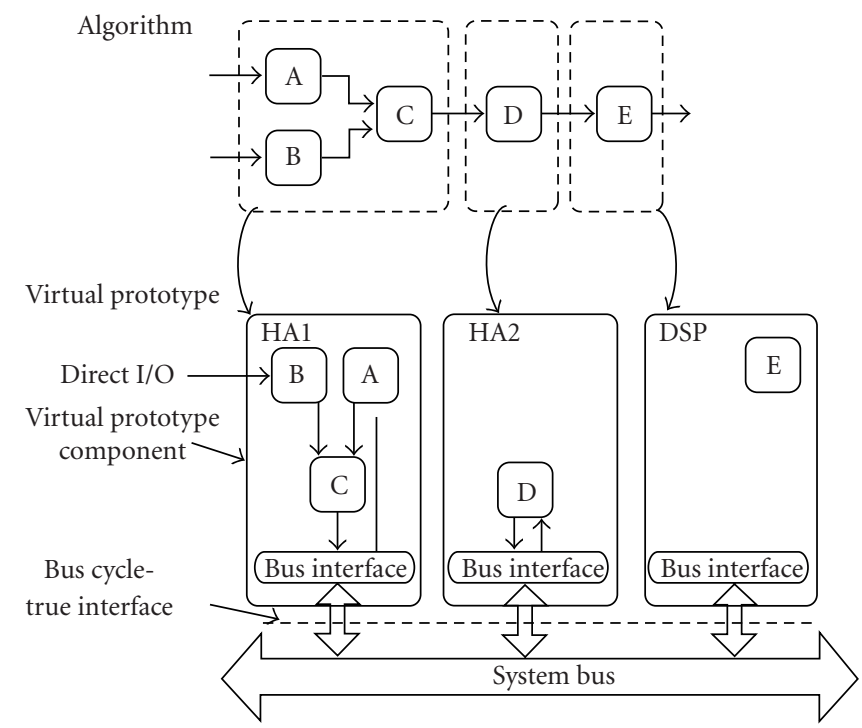

FIGURE 9: Reuse of algorithmic description for virtual prototype generation.

Figure 8. Specific results utilizing this method are reported in Section 6.

A DSP structure enriched by hardware accelerators communicating via a common bus was selected for the design requiring the VP to reflect the DSP as well as to support its hardware accelerators communication (see also Figure 12 further ahead). Note that at the algorithmic level, the design information is free of communication details. Thus, in order to achieve communication of the VP components via the chosen PBD, an object-oriented environment in $\mathrm{C}++$ has been created, containing classes for functional blocks, ports, FIFOs, and scheduling. While this implementation implies a certain HW platform, much emphasis was put on the fact that this platform is very general, a DSP with a common bus structure for its HW accelerator units. The automatism was implemented for COSSAP designs based on GenericC descriptions only. However, the methodology was left open for supporting other descriptions, like SystemC.

In order to provide a quick conversion to $\mathrm{VP}$ as well as short simulation times, it was decided that the VP should not be too detailed (Figure 9). A compromise was made, modeling only the HW/SW interface in cycle-true detail. On the one hand it supports the fast codevelopment of HW and SW engineers and on the other hand simulation times can be 


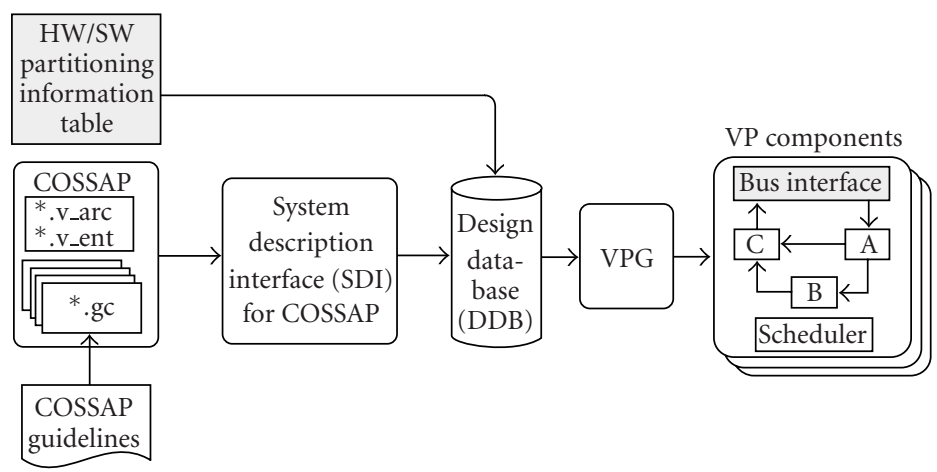

FIGURE 10: Design process for VP generation.

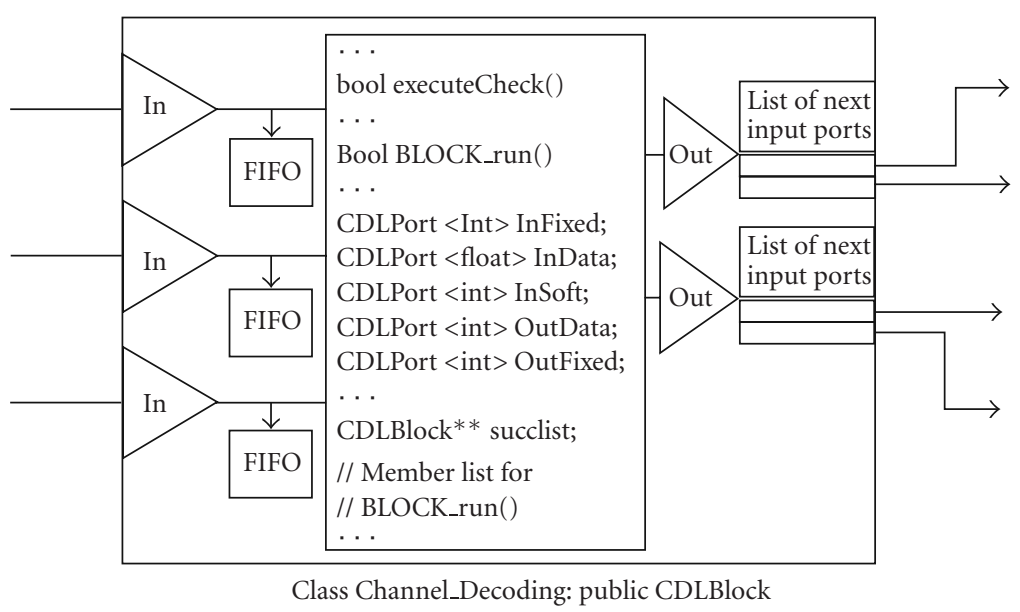

FIGURE 11: A virtual prototype block class.

shortened considerably by reusing the algorithmic description for the DSP accelerators. Such devices are simply modeled by a register interface that can be accessed by the DSP via a common bus. It is thus only necessary to model the processing unit (StarCore-DSP) by an instruction-set simulator (ISS) running the SW part of the design. All other modules later being realized as hardware accelerators are simply copied from the COSSAP description also supporting verification of the design. Moreover, a VP with such properties can serve as a base for prototyping and still be used for further refinements steps towards a whole cycle-true design.

The implementation of such a VP representation needs a simulation environment that allows for simulation of parallel processes. Hardware description languages like SystemC and VHDL can be used for that task, because they provide statements for concurrent processing. For the presented VP another simulation interface has been chosen, that has been proposed by the Virtual Socket Interface Association (VSIA) [17]. This VSIA-compliant simulation uses a static scheduling, achieving faster simulation compared to the event-based simulation of SystemC and VHDL. Even compared to a plain $\mathrm{C}++$ implementation, the VSIA implementation introduces negligible overhead.
Thus as already mentioned in Section 2, some approaches for an automatic partitioning into HW/SW partitioning exist, partitioning in real-world examples is based on much more complicated scenarios even often not based on technical issues. Sometimes implementation in software is preferred in order to stay as long as possible flexible to react to changes in the standard for the implemented algorithm. For this reason the HW/SW partitioning information can be performed manually based on the experience of the various design engineers. This information is manually written into a so-called $H W / S W$ partitioning table (HSP), an extension to the DDB. It is possible to flag each single instance or each instance of a certain module type of the system description as hardware or software (Figure 10). Automatic partitioning embedded in the presented design flow is discussed in [40].

For each module supposed to be implemented in hardware, a $\mathrm{C}++$ file pair, consisting of header and class file, is created automatically. These module classes are derived from the CDLBlock class, which is the centre of the VP component infrastructure. Figure 11 depicts the outline of such a module.

Each derived module instantiates its ports and FIFOs; for each input port a FIFO is provided. The core of each block 
is the "BLOCK_run()." In this procedure the extracted and styled functional part of the GenericC code from a COSSAP project is inserted automatically. Also a $\mathrm{C}++$ file pair for the top module, containing the structure of the VP component, is created automatically. Within the constructor of this class, all instances characterised as HW are instantiated and interconnected.

Furthermore, an additional function is provided to verify that sufficient data for at least one execution of a block is available at the input ports supporting the scheduling capabilities of the VP design. The top module class has a member function acting as a scheduler. Assuming a directed graph structure of the architecture, a scheduler was implemented obeying a recursive depth-first search algorithm [41]. On this account, each block manages a list of its successors which has been set up when the interconnections of all instances were established. The scheduler is responsible for calling each block as often as possible, that is, as long as sufficient data is supplied at the input ports.

Such automatic VP generation requires to restrict the programming freedom of the algorithmic designers. A design style sheet providing guidelines with do's and don'ts was provided to the algorithmic designers. These guidelines include naming conventions for ports, as well as for the corresponding data rates, and internal states of the modules in COSSAP, in order to allow simple and unique automatic recognition. For example, over input ports of each block in the COSSAP design data, values or parameters can be applied, but in the architectural implementation they are handled differently. In order to automate the VP generation it is necessary to give a dedicated prefix to each port name (Input_para or Input_data). According to that naming convention also the names for the port rates require a prefix (Rate_Input_para or Rate_Input_data). The algorithmic designers did not find such guidelines very restricting and quickly adopted their algorithmic design towards it. This step allowed to convert a fixed-point design from algorithmic design level to VP automatically.

Previous implementations of VPs have for the most part focused on their use, in the hardware/software cosimulation of the embedded system $[15,42]$. While these early efforts are targeted towards increasing the efficiency and quality of the design process through novel modifications of the cosimulation process, a transition method (even a manual one) from an algorithmic description to the VP was not shown. A first automatic approach is presented in [14] achieving a speedup in the order of five to eight times compared to a manual VP creation, but it does not support PBDs. In particular, it does not consider the architectural needs of complex SoC, consisting of several hardware accelerators, and a bus system.

\section{CONSISTENT TESTING BY AUTOMATIC TOOLS}

According to $[15,22]$ about $70 \%$ of development time is verification and testing in $3 \mathrm{G}$ wireless designs. Time-consuming parts of testing are the individual test pattern generations performed manually for each design level. It is thus very

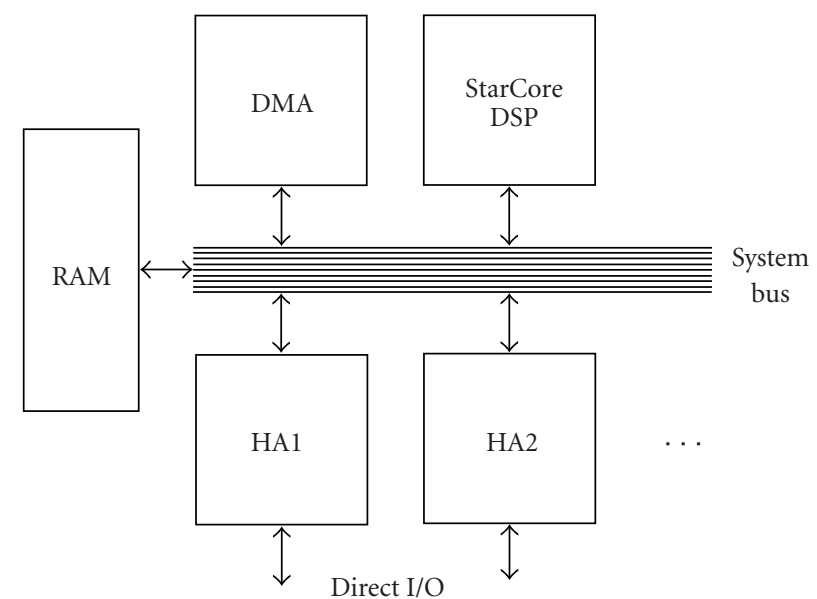

FIgURE 12: Target hardware platform based on a StarCore DSP.

beneficial to convert these test patterns starting from the algorithmic level automatically down to the subsequent levels.

At the VP level, a decision of HW/SW partitioning has been made. The test patterns for the individual blocks have to be provided in a certain order and size as well as in a format specified at the VP design. Thus, the challenge in testing is to provide means for automatically converting high-level test patterns into these specific formats.

\subsection{Overview of hardware platform}

While the testing process in general is rather independent of the HW platform, the actual format is not. Therefore, we first describe more details of the HW platform utilising a StarCore DSP (www.starcore-dsp.com). The hardware platform targeted in this work is a SoC based on a StarCore DSP, as represented in Figure 12.

In addition to the StarCore DSP, the platform includes several hardware accelerators. Just like the DSP, the HA blocks are connected through the system bus to all the other components. Additionally direct I/O interface for HAs are provided, which can be used to connect to components outside the embedded SoC, such as the antenna subsystem. The platform also includes a bank of random access memory (RAM) for use by all the system components. Direct memory access (DMA) services are provided to the DSP, as well as to the HA by a dedicated DMA controller, which is also connected to the system bus.

After HW/SW partitioning is performed on the algorithmic-level description of the system, the algorithmic functions assigned to hardware are implemented as separate HAs, whereas functions assigned to software are realized in software running on the DSP.

\subsection{Reuse of test patterns}

The development of the hardware implementation proceeds in parallel to the development of the other firmware, where both rely on the VP representation. Therefore, it has to be 


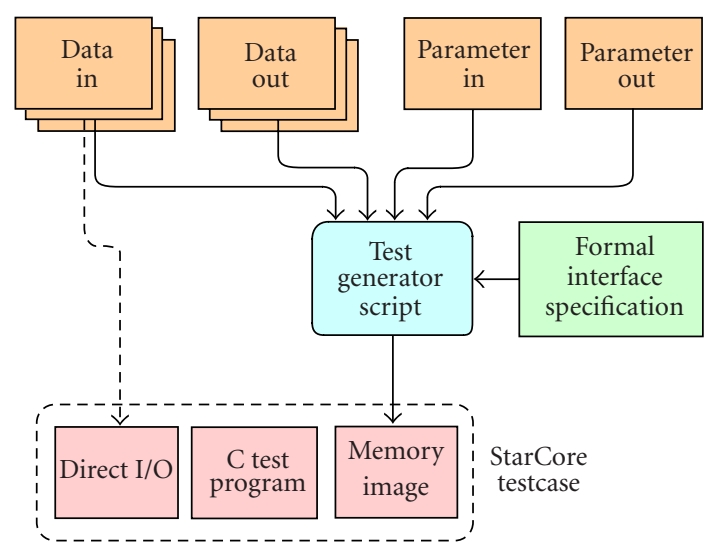

FIGURE 13: Automatic environment for reuse of test patterns.

ascertained that both the VP model and eventually its hardware implementation are functionally equivalent to the original COSSAP model. For this purpose, the automatically generated verification environment presented here reuses all test patters in the COSSAP model and applies them to both the VP and the HW model. Both models exist as part of the same platform (see Figure 12) and for this reason each VP component is tested with the same test bench in both environments.

The automatic environment for reuse of test patterns is shown in Figure 13. The test bench consists of a C program running on the StarCore DSP, a memory image resident in the system's RAM, as well as direct I/O test patterns to be supplied to the VP component during testing. The verification environment includes the automatic generation of test benches immediately from COSSAP test patterns. The inputs into the test generator script (TGS) are a formal specification of the interface between the StarCore DSP and the VP component under test as well as the data and parameter dump files containing the COSSAP test patterns. The output of the test generator script is a test bench, which runs in both the VP simulation environment and on the actual hardware platform.

TGS creates a memory image containing the test patterns, suitably annotated with the timing information (as found in the COSSAP dump files) and the VP component interface information (as found in the formal interface specification). The $\mathrm{C}$ test program is generic and is therefore applicable to any memory image and thus any test bench, without modification. In other words, all case-specific information is captured in the memory image only. When the test patterns in COSSAP include direct I/O data, such data is simply copied into the test bench and applied directly to the VP component through its I/O ports, both in the VP simulation and on the actual HW platform. It is not stored in the memory image, because it is not communicated to the VP component through the system bus and is thus not handled by the $\mathrm{C}$ test program.

\subsection{The VP as golden model for the HW}

At the time the VP has been automatically created and its functionality at the bus interface has been tested using the verification environment explained in the section before, it can be further used as a golden model for the HW development.

The VP component organises the synchronous data flow between its inner functional components using a FIFO class. In order to provide a verification facility while running a VP simulation, the implementation of the FIFO blocks includes a bi-directional insertion/extraction feature. In other words, in addition to simply being passed from one functional block to another, data probes can be handed to output (dump mode) files as well as read from input files (pump mode) independently. This file input/output functionality exists in every FIFO in the VP model and thus offers maximum flexibility in inspecting and/or inserting test patterns at any point within the VP structure, depicted in Figure 14. Provided that the HW developer organises the internal HW accelerator design in the same functional fragmentation (e.g., a corresponding VHDL entity for each $\mathrm{C}++$ class in the VP component), the test patterns generated by the VP blocks can be adopted to verify the proper functionality of the VHDL entities.

Note that the fragmentation into several blocks in the VP component is very coarse as it mirrors the highest abstraction level, the algorithmic description in COSSAP. Thus the impact on the creativity of the HW developer with respect to design and optimisation issues at the RT-level is negligible. Experience from HW development (by industrial collaboration) revealed that the demand for test patterns at predefined points is much higher than the demand for less structural requirements.

As mentioned before the internal representation of the VP component is generally untimed to offer highest simulation speed, just in opposition to the cycle-true HW implementation. Therefore, the data probes from the VP cannot contain timing information below the bus-cycle accurate level. Since the sequence of the values, sent from one internal block to the next, remains unaffected, the verification of the corresponding VHDL entities is enabled. A file format has been defined which captures the necessary information to identify the source block, the port name, the bus cycle, and the numbering of the generated value. A convenient way to analyse the corresponding test pattern files is via the Open Source tool TkDiff (http://sourceforge.net/projects/tkdiff/), offering graphical support for the comparison of text files.

\section{EXAMPLES OF UMTS DESIGNS}

According to the design flow presented in Section 4, the entire COSSAP project for a UMTS baseband processing unit (UMTS BPU) has been analysed by the SDI and imported to the DDB. After partitioning of the algorithmic description of the UMTS BPU into SW and HW components, ten VP peripherals have been generated. Four of them assemble the UMTS cell phone receiver part and will be discussed 


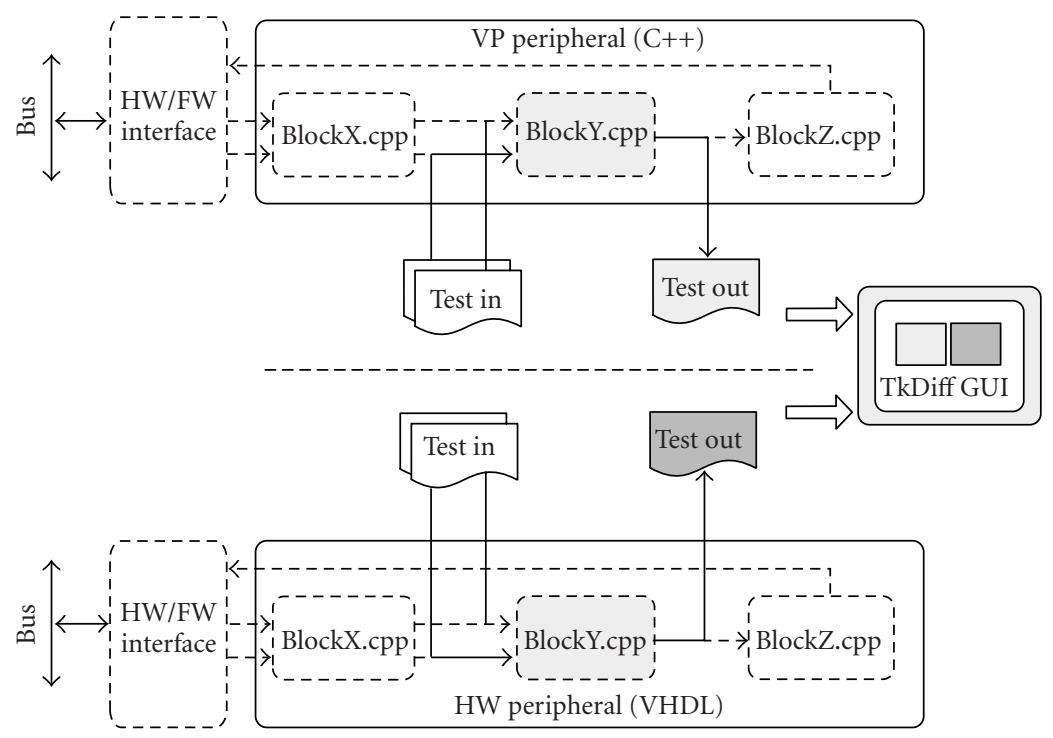

FIGURE 14: VP as a golden model for HW implementation.

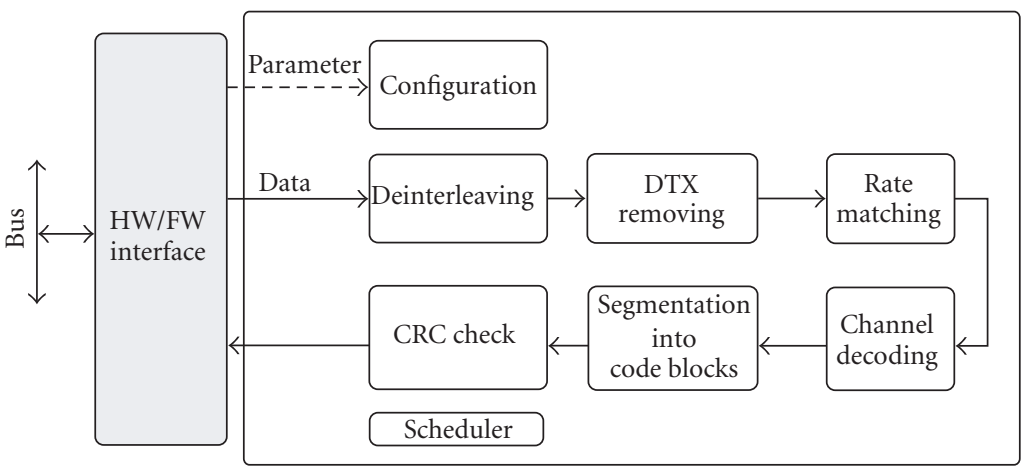

Class DUD: public CDLVp

FIGURE 15: VP block diagram of the peripheral for decoding of user data (DUD).

more thoroughly in the following. Each of the four parts realizes the complete or partial functionality of (a) delay profile estimation (DPE), (b) frame and slot synchronisation (SYNC), (c) rake receiver (RAKE), and (d) decoding of user data (DUD). As a detailed example, Figure 15 depicts the DUD peripheral to expose the VP infrastructure of the automatically generated HW peripheral.

This DUD consists of six blocks, representing the digital signal processing for the decoding of user data according to 3GPP TS25.212, and additional three blocks (configuration, $H W / F W$-interface, and scheduler) generated by the VP in order to support scheduling. The configuration block collects the initial parameters of the six signal processing blocks, which cannot be passed by the HW/FW interface during run-time and delivers them to each block to set up the specific behaviour, for instance switching the data processing from a slot to a frame basis. Note that the COSSAP developer has the possibility to change the parameters of a block before each block invocation. In the VP as well as in the real HW implementation this is different; it is only possible to update the parameters of the blocks between two peripheral invocations. The $H W / F W$ interface communicates via the on-chip bus with the StarCore DSP, configures the DUD peripheral, fills the FIFOs of the deinterleaving block with data and triggers the scheduler function.

To achieve the time savings due to the presented automation, it was necessary to port the HW peripherals manually from the COSSAP project to the VP representation (except for the RAKE receiver because of its extraordinary size). Within this process the expenditure of human labour, measured in person hours $(\mathrm{PH})$, for translating the behavioural model of each module into a $\mathrm{C}++$ file pair has been documented. In order to predict the time expenditure of the remaining blocks, we assume the time expense for each task is proportional to the lines of code (LOC) of the GenericC files of the corresponding modules. The results are listed in Table 1, showing also that the efficiency (avg. PH/LOC $=0.017$ ) of the manual work is roughly constant for each module. 
TABLE 1: Algorithmic complexity and time expense of the manually generated VP peripherals $(\mathrm{PH}=$ person hour, $\mathrm{LOC}=$ lines of code $)$.

\begin{tabular}{c|ccc}
\hline HW peripheral & LOC & PH & PH/LOC \\
\hline DPE & 1653 & 25 & 0.015 \\
SYNC & 2422 & 39 & 0.016 \\
DUD & 2398 & 43 & 0.018
\end{tabular}

TABLE 2: Infrastructural complexity and time expense of the manually generated VP peripherals $(\mathrm{PH}=$ person hour, $\mathrm{NIB}=$ number of instantiated blocks).

\begin{tabular}{c|ccc}
\hline HW peripheral & NIB & PH & PH/NIB \\
\hline DPE & 5 & 8 & 1.60 \\
SYNC & 7 & 17 & 2.43 \\
DUD & 6 & 12 & 2.00 \\
\hline
\end{tabular}

In a second step, the creation of the $\mathrm{C}++$ files for the top module, which instantiates, interconnects, and configures the blocks, has been accomplished. The time spent is assumed to be proportional to the number of instantiated blocks (NIB), further on referred to as infrastructural complexity. The results are listed in Table 2 showing an avg. $\mathrm{PH} / \mathrm{NIB}=2.0$.

As the HW/FW interface has to be described manually with respect to the underlying architecture, its development time is not taken into account for the evaluation of time reduction. Assuming that the averaged values of the PH/LOC and $\mathrm{PH} / \mathrm{NIB}$ ratios obtained from UMTS receiver are reliable metrics for the remaining peripherals of the whole UMTS $\mathrm{BPU}$, it is possible to estimate the design effort for the manual generation of all VP peripherals. An amount of $774 \mathrm{PH}$ for 121 NIBs and 31266 LOCs was estimated. Employing the presented automatic approach, each of the VP peripherals is generated within seconds.

Note that in an industrial development such mapping from the fixed-point algorithmic model to the VP model is not performed just once. Many revision levels are required until the functionality of all modules is satisfactory. Now, in the middle of the design process, our development routine revealed revision levels for the peripherals of already more than fifty; and at each revision version, in which especially the infrastructure (e.g., number of ports, connections) has been changed to a certain degree, this approach exposes its superior performance, applying the automatic mapping procedure. The time savings accumulate to many thousand person hours over an entire design. Its application in an industry project, where an ISS of a StarCore together with the debugging facilities of the CodeWarrior environment from Metrowerks has been used, proved that the modification of an existing COSSAP project necessary to be compliant to the GenericC guidelines causes negligible effort, while the speedup of development time is substantially. Note, however, that this simple arithmetic does not show the only benefit of the proposed method. A manual process would have consumed not only thousands of additional hours but also many more hours required to find the manually generated bugs, whereas an exhaustively tested tool is almost error free.

\section{CONCLUSIONS}

The automated environment for VP generation presented here has been successfully applied in an industrial design flow, showing significant speedup in creation of VPs, with savings in the order of hundreds of person hours per revision. Simultaneously, this approach also eliminates human-related errors, thus improving quality. Additionally, work presented here shows better performance benefits, increased flexibility, and wider applicability compared to previously presented automated techniques. Future work on the presented environment includes generation of VPs in standards other than VSIA, such as SystemC, CoMET from Vast System Technology (www.vastsystems.com), or SEAMLESS from Mentor Graphics (www.mentor.com). Also processing of algorithmic descriptions developed in environments other than COSSAP are of interest. Furthermore a direct binding of the presented VP environment with more of the numerous commercial algorithmic- and architecture-level tools is expected to increase automation and thus significantly gain design efficiency.

\section{ACKNOWLEDGMENTS}

The authors would like to acknowledge the ongoing cooperation with Infineon Technologies and in particular thank Thomas Herndl, Ahmad Sarashgi, Wolfgang Haas, Sasha Simeunovic, and Johann Glaser for their collaboration. This work has been funded by the Christian Doppler Laboratory for Design Methodology of Signal Processing Algorithms.

\section{REFERENCES}

[1] G. Moore, "Cramming more components onto integrated circuits," Electronics Magazine, vol. 38, no. 8, pp. 114-117, 1965.

[2] P. Fisher and D. Cottrell, "Emerging standards in the electronic design automation (EDA) industry," in Electronic Systems Design Seminar, UC Berkeley, Calif, USA, October 1999.

[3] R. Subramanian, "Shannon vs Moore: the digital signal processing in the broadband age," in Proc. IEEE Communication Workshop, Aptos, Calif, USA, May 1999.

[4] R. Subramanian, "Shannon vs Moore: driving the evolution of signal processing platforms in wireless communications," in Proc. IEEE Workshop on Signal Processing Systems (SIPS '02), San Diego, Calif, USA, October 2002.

[5] P. Belanović, M. Holzer, D. Mičušík, and M. Rupp, "Design methodology of signal processing algorithms in wireless systems," in Proc. International Conference on Computer, Communication and Control Technologies (CCCT '03), pp. 288291, Orlando, Fla, USA, July 2003.

[6] M. Holzer, P. Belanović, and M. Rupp, "A consistent design methodology to meet SDR challenges," in Wireless World Research Forum (WWRF9 '03), Zurich, Switzerland, July 2003.

[7] R. Baines and D. Pulley, "A total cost approach to evaluating different reconfigurable architectures for baseband processing in wireless receivers," IEEE Commun. Mag., vol. 41, no. 1, pp. 105-113, 2003.

[8] R. Leupers and P. Marwedel, "Retargetable code generation based on structural processor descriptions," Design Automation for Embedded Systems, vol. 3, no. 1, pp. 1-36, 1998.

[9] J. Brakensiek, B. Oelkrug, M. Bucker, et al., "Software radio approach for re-configurable multi-standard radios," in Proc. 13th IEEE International Symposium on Personal, Indoor and 
Mobile Radio Communications (PIMRC '02), vol. 1, pp. 110 114, Lisboa, Portugal, September 2002.

[10] P. M. Heysters, H. Bouma, J. Smit, G. J. M. Smit, and P. J. M. Havinga, "A reconfigurable function array architecture for $3 \mathrm{G}$ and 4G wireless terminals," in Proc. World Wireless Congress (WWC '02), pp. 399-405, San Francisco, Calif, USA, May 2002.

[11] J. R. Hauser and J. Wawrzynek, "Garp: a MIPS processor with a reconfigurable coprocessor," in Proc. 5th Annual IEEE Symposium on FPGAs for Custom Computing Machines (FCCM '97), pp. 12-21, Napa Valley, Calif, USA, April 1997.

[12] M. B. Taylor, J. Kim, J. Miller, et al., "The Raw microprocessor: a computational fabric for software circuits and generalpurpose programs," IEEE Micro, vol. 22, no. 2, pp. 25-35, 2002.

[13] C. A. Valderrama, A. Changuel, and A. A. Jerraya, "Virtual prototyping for modular and flexible hardware-software systems," Design Automation for Embedded Systems, vol. 2, no. 34, pp. 267-282, 1997.

[14] A. Hemani, A. K. Deb, J. Oberg, A. Postula, D. Lindqvist, and B. Fjellborg, "System level virtual prototyping of DSP SOCs using grammar based approach," Design Automation for Embedded Systems, vol. 5, no. 3-4, pp. 295-311, 2000.

[15] A. Hoffmann, T. Kogel, and H. Meyr, "A framework for fast hardware-software co-simulation," in Proc. Design, Automation and Test in Europe (DATE '01), pp. 760-765, Munich, Germany, March 2001.

[16] N. Voros, L. Sánchez, A. Alonso, A. Birbas, M. Birbas, and A. Jerraya, "Hardware/software co-design of complex embedded systems: an approach using efficient process models, multiple formalism specification and validation via co-simulation," Design Automation for Embedded Systems, vol. 8, no. 1, pp. 549, 2003.

[17] U. Bortfeld and C. Mielenz, "C++ system simulation interfaces," Whitepaper, July 2000.

[18] R. Ernst, J. Henkel, and T. Benner, "Hardware-software cosynthesis for microcontrollers," IEEE Des. Test. Comput., vol. 10, no. 4, pp. 64-75, 1993.

[19] T. Wiangtong, P. Y. K. Cheung, and W. Luk, "Comparing three heuristic search methods for functional partitioning in hardware-software codesign," Design Automation for Embedded Systems, vol. 6, no. 4, pp. 425-449, 2002.

[20] T. Blickle, J. Teich, and L. Thiele, "System-level synthesis using evolutionary algorithms," Design Automation for Embedded Systems, vol. 3, no. 1, pp. 23-58, 1998.

[21] CoWare, "SoC platform-based design using ConvergenSC/SystemC," July 2002, http://www.coware.com.

[22] B. Bailey, "The Waking of the Sleeping Giant-Verification," April 2002, http://www.mentor.com/consulting/techpapers/.

[23] U. Glässer, R. Gotzhein, and A. Prinz, "The formal semantics of SDL-2000: Status and perspectives," Computer Networks, vol. 42, no. 3, pp. 343-358, 2003.

[24] T. Grötker, S. Liao, G. Martin, and S. Swan, System Design with SystemC, Kluwer Academic, Boston, Mass, USA, 2002.

[25] M. Coors, H. Keding, O. Lüthje, and H. Meyr, "Design and DSP implementation of fixed-point systems," EURASIP J. Appl. Signal Process., vol. 2002, no. 9, pp. 908-925, 2002.

[26] M. Rupp, A. Burg, and E. Beck, "Rapid prototyping for wireless designs: the five-ones approach," Signal Processing, vol. 83, no. 7, pp. 1427-1444, 2003.

[27] F. Balarin, E. Sentovich, M. Chiodo, et al., Hardware-Software Co-Design of Embedded Systems: The POLIS Approach, Kluwer Academic, Boston, Mass, USA, 1997.
[28] G. Berry, S. Moisan, and J. Rigault, "ESTEREL: Towards a synchronous and semantically sound high level language for real-time applications," in Proc. IEEE Real-Time Systems Symposium (RTSS '83), pp. 30-37, IEEE Computer Society Press, Arlington, Va, USA, December 1983.

[29] N. Halbwachs, P. Caspi, P. Raymond, and D. Pilaud, "The synchronous data flow programming language LUSTRE," Proc. IEEE, vol. 79, no. 9, pp. 1305-1320, 1991, Special Issue "Another Look at Real-Time Programming".

[30] J. Buck, S. Ha, E. A. Lee, and D. G. Messerschmitt, "Ptolemy: a framework for simulating and prototyping heterogeneous systems," International Journal in Computer Simulation, vol. 4, no. 2, pp. 155-182, 1994, Special Issue on Simulation Software Development.

[31] J. Sztipanovits, "Advances in model-integrated computing," in Proc. 18th IEEE Instrumentation and Measurement Technology Conference (IMTC'01), vol. 3, pp. 1660-1664, Budapest, Hungary, May 2001.

[32] G. Karsai, J. Sztipanovits, A. Ledeczi, and T. Bapty, "Modelintegrated development of embedded software," Proc. IEEE, vol. 91, no. 1, pp. 145-164, 2003.

[33] J. Gray and G. Karsai, "An examination of DSLs for concisely representing model traversals and transformations," in Proc. 36th Annual Hawaii International Conference on System Sciences (HICSS '03), vol. 9, Big Island, Hawaii, USA, January 2003.

[34] G. Karsai, "Design tool integration: an exercise in semantic interoperability," in Proc. 7th IEEE International Conference and Workshop on the Engineering of Computer Based Systems (ECBS '00), pp. 272-278, Edinburgh, UK, April 2000.

[35] J. Groff and P. Weinberg, SQL: The Complete Reference, McGraw-Hill, Osborne, Emeryville, Calif, USA, 2nd edition, 2002.

[36] L. Cai and D. Gajski, "Transaction level modeling in system level design,” Tech. Rep., Center for Embedded Computer Systems, Irvine, Calif, USA, 2003.

[37] A. Haverinnen, M. Leclercq, N. Weyrich, and D. Wingard, "White Paper: SystemC based SoC Communication Modeling for the OCP Protocol," OCP International Partnership, Portland, Ore, USA, October 2002.

[38] S. Gupta, N. Dutt, R. Gupta, and A. Nicolau, "SPARK: A highlevel synthesis framework for applying parallelizing compiler transformations," in Proc. 16th International Conference on VLSI Design, pp. 461-466, New Delhi, India, January 2003.

[39] W. Wu and A. Jantsch, "A survey of design transformation techniques," Tech. Rep., Departement of Electronics, Royal Institute of Technology, Kista, Sweden, 1999. http://www.ida.liu.se/ eslab/SAVE/.

[40] B. Knerr, M. Holzer, and M. Rupp, "HW/SW partitioning using high level metrics," in Proc. International Conference on Computing, Communications and Control Technologies (CCCT '04), pp. 33-38, Austin, Tex, USA, August 2004.

[41] R. Sedgewick, Algorithms, Addison-Wesley, Boston, Mass, USA, 1988 .

[42] J. Cockx, "Efficient modeling of preemption in a virtual prototype," in Proc. 11th International Workshop on Rapid System Prototyping (RSP '00), pp. 14-19, Paris, France, June 2000. 
P. Belanović received his B.E. and M.S. degrees from the University of Auckland, New Zealand, and Northeastern University, Boston, in 2000 and 2002, respectively. His research focused on the acceleration of image processing algorithms with reconfigurable platforms, both in remote sensing and biomedical domains, as well as customformat floating-point arithmetic. Currently he is a Ph.D. candidate at the Vienna Uni-

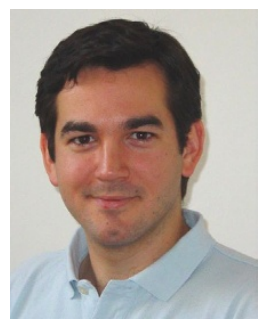
versity of Technology, Austria, focusing on the design methodologies for embedded systems in wireless communications, virtual prototyping, and automated floating-point to fixed-point conversion.

B. Knerr studied communications engineering at the University of Saarland and the Technical University of HamburgHarburg, respectively. He finished the Diploma thesis about OFDM communications systems and graduated with honours in 2002. He worked for one year as a software engineer for the UZR GmbH \& Co KG, Hamburg, on image processing and 3D computer vision. In June 2003 he joined the

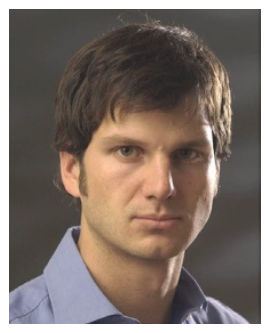
Christian Doppler Laboratory for Design Methodology of Signal Processing Algorithms at the Vienna Technical University as a Ph.D. candidate. His research interests are in HW/SW partitioning, multicore task scheduling, static code analysis, and platform-based design.

M. Holzer received his Dipl.-Ing. degree in electrical engineering from the Vienna University of Technology, Austria, in 1999. During his Diploma studies, he worked on the hardware implementation of the LonTalk protocol for Motorola. From 1999 to 2001 he worked at Frequentis in the area of automated testing of TETRA systems and afterwards until 2002 at Infineon Technologies on ASIC design for UMTS mobiles. Since

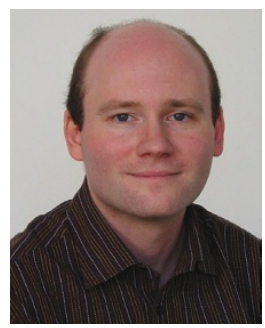
2002 he has a research position at the Christian Doppler Laboratory for Design Methodology of Signal Processing Algorithms at the Technical University of Vienna.

G. Sauzon received his Engineer degree from ENSERG, Grenoble, France. He joined Infineon Technologies in 1999, focusing on the architecture definition of a UMTS receiver. His work also included analysis of design flows and development of virtual prototypes for architecture verification. His current interests are focused on verification of baseband processors.

M. Rupp received his Dipl.-Ing. degree in 1988 from the University of Saarbrücken, Germany, and his Dr.-Ing. degree in 1993 from the Technische Universität Darmstadt, Germany, where he worked with Eberhardt Hänsler on designing new algorithms for acoustical and electrical echo compensation. From November 1993 until July 1995 he had a postdoctoral position at the
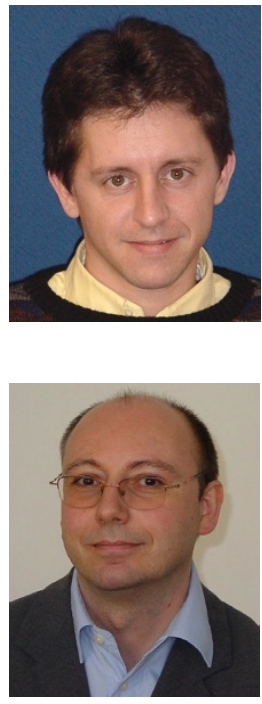

University of Santa Barbara, California, with Sanjit Mitra where he worked with Ali $\mathrm{H}$. Sayed on a robustness description of adaptive filters with impacts on neural networks and active noise control. From October 1995 until August 2001 he was a member of the technical staff in the Wireless Technology Research Department of Bell-Labs where he was working on various topics related to adaptive equalization and rapid implementation for IS-136, 802.11, and UMTS. He is presently a Full Professor for digital signal processing in mobile communications at the Technical University of Vienna. $\mathrm{He}$ is an Associate Editor of the IEEE Transactions on Signal Processing and of EURASIP JASP and is an elected AdCom Member of EURASIP. He authored and coauthored more than 100 papers and patents on adaptive filtering, wireless communications, and rapid prototyping. 